\title{
ENSAIO DE IMPACTO CHARPY EM MATRIX EPOXÍDICA REFORÇADA COM TECIDO DE FIQUE*
}

\author{
Maria Carolina Andrade Teles ${ }^{1}$ \\ Marcos Vinícius Fonseca Ferreira ${ }^{1}$ \\ Sérgio Neves Monteiro ${ }^{2}$ \\ Felipe Perissé Duarte Lopes ${ }^{3}$ \\ Djalma Souza ${ }^{4}$
}

\section{Resumo}

Nas últimas décadas, questões ambientais estão motivando novos paradigmas para o desenvolvimento de nossa sociedade. Assim, os compósitos epoxídicos reforçados com fibras lignocelulósicas atraíram o interesse em várias áreas de engenharia devido ao seu baixo custo, baixo consumo de energia durante a fabricação em associação com várias aplicações possíveis. A fibra, que é avaliada no presente trabalho, é extraída da folha da planta do fique (Furcraea Andina), demonstra um grande potencial como reforço de compósitos poliméricos. Este trabalho avaliará o desempenho em ensaio de impacto Charpy, de compósitos com matriz epóxi associada a diferentes camadas de tecido de fibras de Fique. Os resultados mostraram um aumento notável na dureza do entalhe com a quantidade de camadas de tecido de Fique incorporadas. Isso pode ser atribuído a uma desvinculação preferencial da interface fibra / matriz, o que contribui para uma energia absorvida elevada.

Palavras- Chave: Compósitos; Tecido de Fique; Resina Epóxi; Impacto Chapy.

\section{IMPACT CHARPY TEST IN EPOXY MATRIX REINFORCED WITH FIQUE FABRIC}

\section{Abstract}

In recent decades, environmental issues are motivating new paradigms for the development of our society. Epoxy composites reinforced with lignocellulosic fibers have attracted interest in several engineering areas due to their low cost, low energy consumption during fabrication in association with a number of possible applications. The fiber, which is evaluated in the present work, is extracted from the leaf of fique plant (Furcraea Andina), demonstrates a great potential as reinforcement of polymeric composites. This work will evaluate the performance of impact charpy test in composites with epoxy matrix associated to different fabric layers of fique fibers. The results showed an increase in the toughness with amount of fique fabric. Attributed to a weak resistance of fiber/matrix interface, which contributes to an elevated absorbed energy.

Keywords: Composites; Fique Fabric; Epoxy; Impact Chapy.

1 Graduando(a) em Engenharia Metalúrgica e de Materiais, bolsista de iniciação científica, LAMAV, UENF, Campos dos Goytacazes, Rio de Janeiro - Brasil.

2 Engenheiro Metalúrgico, Doutor em Ciência e Engenharia de Materiais, Professor titular, IME, Rio de Janeiro, Rio de Janeiro - Brasil.

3 Engenheiro Metalúrgico, Doutor em Ciência e Engenharia de Materiais

4 Engenheiro de Metalúrgico, Doutor em Ciências de Materiais, Livre-Docência, LAMAV, UENF, Campos dos Goytacazes, Rio de Janeiro - Brasil.

\section{INTRODUÇÃO}


As tecnologias atuais vêm exigindo cada vez mais materiais que possuam propriedades atípicas que não podem ser atendidas pelas ligas metálicas, materiais poliméricos ou cerâmicos. A indústria aeronáutica, por exemplo, tem buscado materiais que sejam fortes, rígidos, apresentem elevada resistência à abrasão e ao impacto, de baixa densidade e resistentes à corrosão. $O$ desenvolvimento de materiais compósitos, através de uma combinação conciliada de dois ou mais materiais distintos gera propriedades consideravelmente formidáveis, atendendo às demandas de indústrias aeroespaciais, subaquáticas e de transporte.

No quesito tecnologia, os compósitos reforçados com a fase dispersa em forma de fibra são os mais relevantes [1]. As fibras naturais estão substituindo constantemente fibras sintéticas, particularmente a fibra de vidro comum, como a fase de reforço de compósitos poliméricos em muitas aplicações de engenharia como componentes interiores de automóvel, capacetes para ciclistas, painéis de carcaça entre outros [2-4]. O processamento para a fabricação de fibra de vidro, está associado à emissão de $\mathrm{CO}_{2}$, e a dificuldade em reciclar resíduos produtos do processo[5,6].

Além dos benefícios ambientais [7,8], as fibras lignocelulósicas apresentam vantagens econômicas como a abundância mundial e custos comparativamente baixos, bem como algumas propriedades técnicas associadas à flexibilidade e tenacidade [9], que contribuem para o desempenho de componentes automotivos fabricados com compósitos de fibras naturais [4]. Adicionalmente, as fibras naturais são recicláveis, biodegradáveis, possuem baixa densidade e não emitem $\mathrm{CO}_{2}$ durante seu processo de fabricação.

A planta de Fique (Furcraea Andina) é nativa dos Andes do Equador Colômbia e Venezuela, porém difundida em andes colombianos. A fibra é resistente, fina, branca e brilhante. A produção normal, anual, é de $1 \mathrm{~kg}$ por planta, com produções excepcionais de 3 a $6 \mathrm{~kg}$ por ano [10]. Como as características heterogêneas das fibras lignocelulósicas, isso é uma limitação para sua utilização em compósitos, o presente trabalho realizou a análise do comportamento mecânico na resistência à impacto na configuração Charpy do compósito polimérico de epóxi reforçado com tecido de Fique.

\section{MATERIAIS E MÉTODOS}

Os materiais usados no presente trabalho foram a resina epóxi (DGEBA) já misturada com o endurecedor em estequiometria, phr 13, trietilenotetramina (TETA) e as fibras extraídas da folha da planta Fique, fornecidas por seus produtores da Colômbia. As fibras usadas foram fornecidas na configuração de tecido, Fig. 2. 


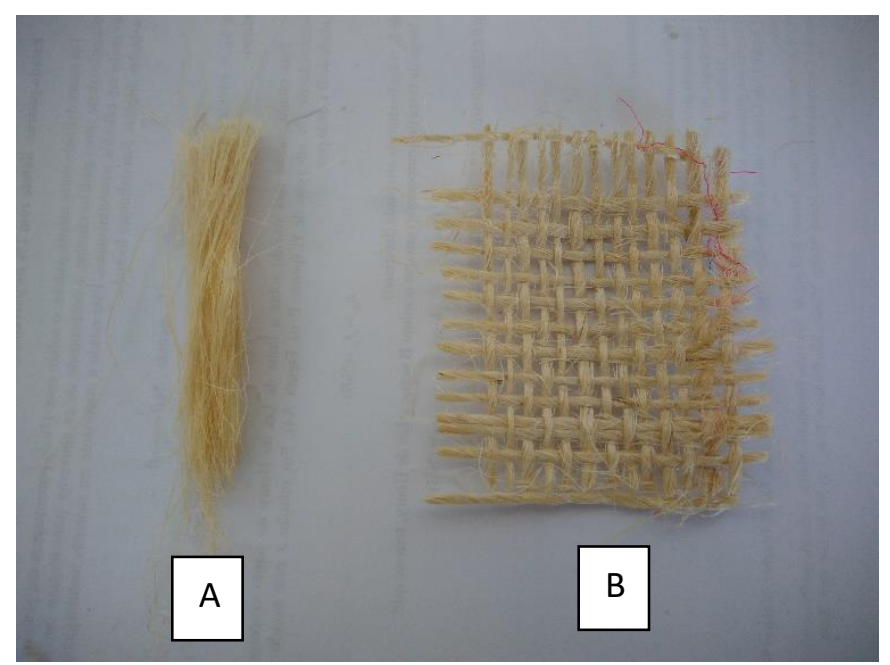

Figura 1: (a) planta de fique e (b) tecido da fibra de fique.

Os compósitos com 1, 3, 4, 5 e 6 camadas de tecido foram fabricados através da acomodação do tecido junto à resina ao molde de aço. $O$ molde foi colocado para cura sob uma pressão de 5 toneladas à temperatura ambiente durante 24 horas. As placas utilizadas na preparação de corpos de impacto Charpy foram cortadas com dimensões de $120 \times 12 \times 10 \mathrm{~mm}$ de acordo com a ASTM D6110 [11], totalizando 9 corpos de prova para cada placa. Os corpos de prova, com entalhe de 2,54 $\mathrm{mm}$ de profundidade e 45 graus de abertura, foram ensaiados em um pêndulo PANTEC, modelo XC - 50 com a configuração Charpy, Fig. 2. O modelo de corpo de prova com as dimensões devidas é mostrado na Fig.3.

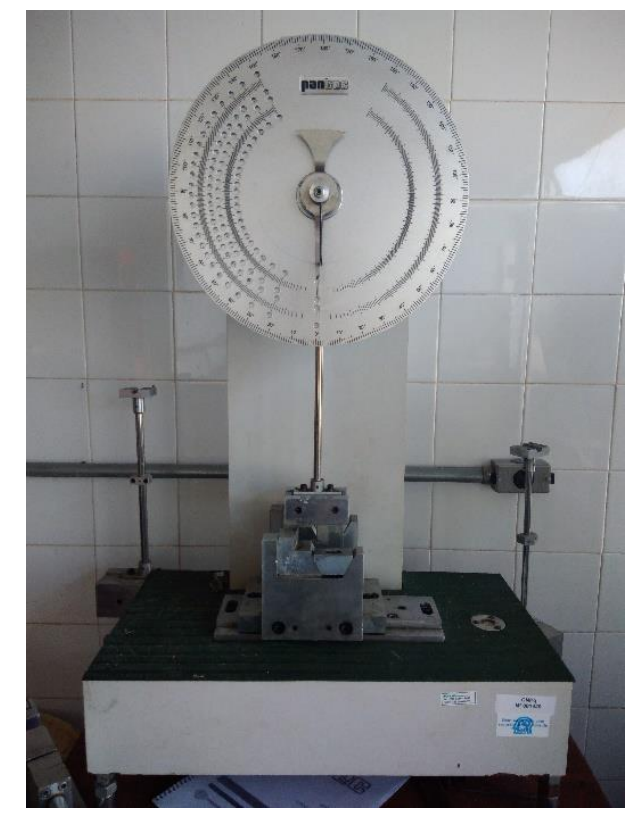

Figura 2: Martelo PANTEC utilizado no ensaio de Impacto Charpy. 


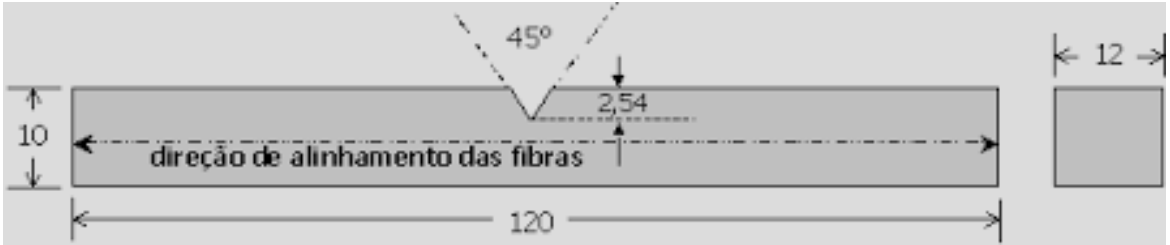

Figura 3: Corpo de prova padrão para o ensaio de impacto ,tipo charpy.

\section{RESULTADOS E DISCUSSÕES}

A Tabela I mostra os resultados dos valores da energia de impacto Charpy com seus respectivos desvios padrões para epóxi puro e compósitos com diferentes camadas e frações volumétricas de tecido fibra fique.

Tabela 1 : Resultados da Energia de Impacto absorvida com o aumento do número de camadas de Tecido de Fique.

\begin{tabular}{|c|c|c|}
\hline $\begin{array}{c}\text { Número de } \\
\text { Camadas de } \\
\text { Tecido }\end{array}$ & $\begin{array}{c}\text { Fração } \\
\text { Volumétrica } \\
\text { de Fibras }(\%)\end{array}$ & $\begin{array}{c}\text { Energia de } \\
\text { Impacto } \\
\text { Charpy }(\mathrm{J} / \mathrm{m})\end{array}$ \\
\hline 0 & 0 & $25,3 \pm 1,6$ \\
\hline 1 & 6,2 & $96,7 \pm 12,3$ \\
\hline 2 & 19,4 & $200,3 \pm 26,9$ \\
\hline 3 & 23,9 & $285,9 \pm 47,8$ \\
\hline 4 & 29,6 & $318,5 \pm 61,2$ \\
\hline 5 & 34,1 & $348,4 \pm 70,2$ \\
\hline
\end{tabular}

Com base nos resultados apresentados na Tabela 1, a variação da energia de impacto de Charpy com a quantidade de tecido de fique no compósito de epóxi é mostrado na Fig. 4.

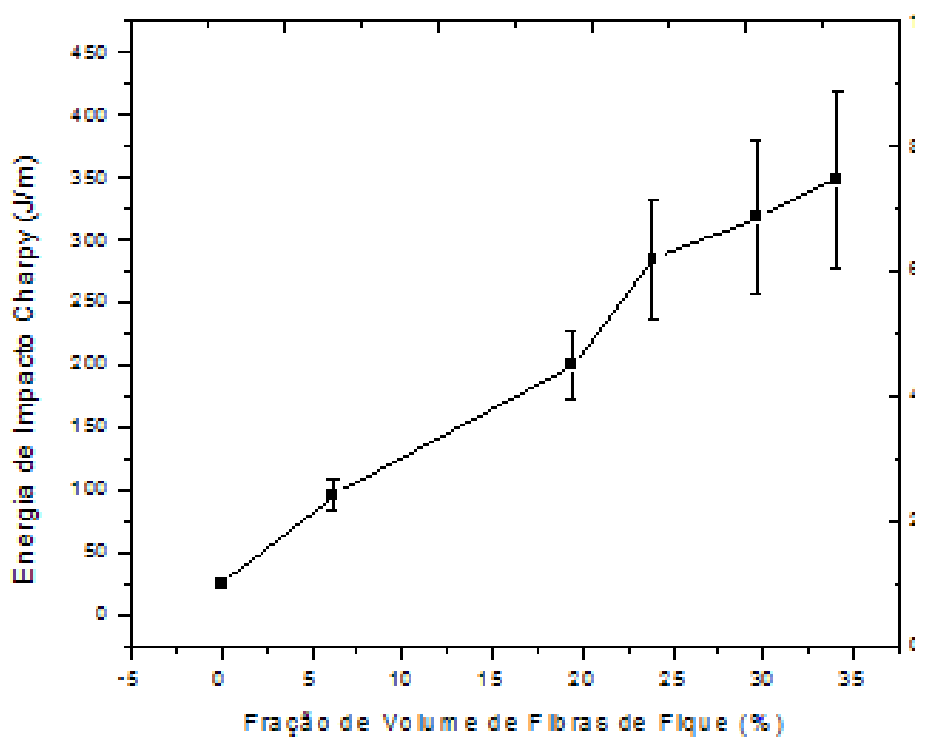


Figura 4: Energia de impacto de Charpy em função do número de camadas de tecido de fique.

De fato, a incorporação de tecido de fique aumenta consideravelmente a energia de impacto absorvida, quando comparada à matriz somente com a resina epóxi, atingindo um valor 100 vezes maior de energia. A alta dispersão de valores já era esperada haja vista que, é um característica heterogênea das fibras lignocelulósicas[12]. Outro ponto importante a ser destacado é o fato da matriz polimérica ser hidrofóbica e a fibra ser hidrofílica. Esse fato resulta em uma baixa interface fibra/matriz, contribuindo para uma transferência de carga parcialmente ineficaz. O aspecto macroscópico dos corpos de prova após ruptura devido ao ensaio de Impacto, é mostrado na Fig. 5.

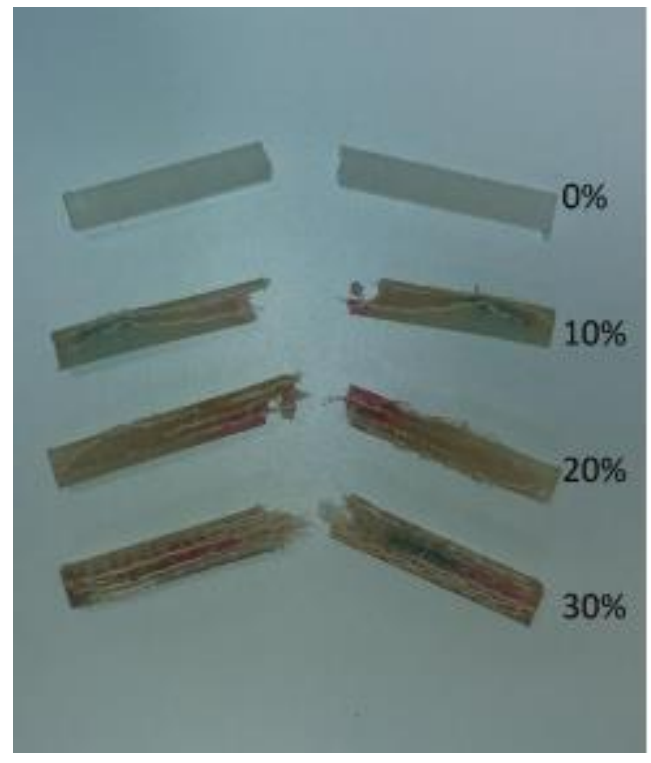

Figura 5: Aspecto macroscópico dos corpos de prova rompidos

Nota-se que até com 4 camadas de tecido, os corpos de prova se romperam, sendo totalmente separados após o ensaio. A partir de 5 camadas, algumas amostras não se desprenderam inteiramente após o impacto do martelo Charpy de $11 \mathrm{~J}$, e assim, esses resultados não podem ser comparados com os resultados dos corpos rompidos por completo.

Além disso, pode-se também observar na Fig. 5 que, a ruptura sempre ocorre no entalhe e que, a adição de camadas de tecido de Fique, mesmo com apenas 1 camada, resulta em uma ruptura que não é completamente transversal. Esse aspecto macroscópico indica que trincas iniciadas no entalhe se propagam transversalmente através da matriz de epóxi, e quando a trinca atinge uma fibra, a ruptura irá prosseguir através da interface.

Para melhor entendimento do mecanismo responsável pelo aumento de tenacidade dos compósito de epóxi reforçados com tecido de Fique, foi feita uma análise de MEV na fratura dos corpos de prova. A Fig.6 mostra que, ao encontrar as fibras, a trinca sofre desvio. Além disso, as fibras que participaram da ruptura, têm aproximadamente a mesma altura que a matriz, confirmando novamente que o deslizamento entre a matriz e a fibra não ocorre. 

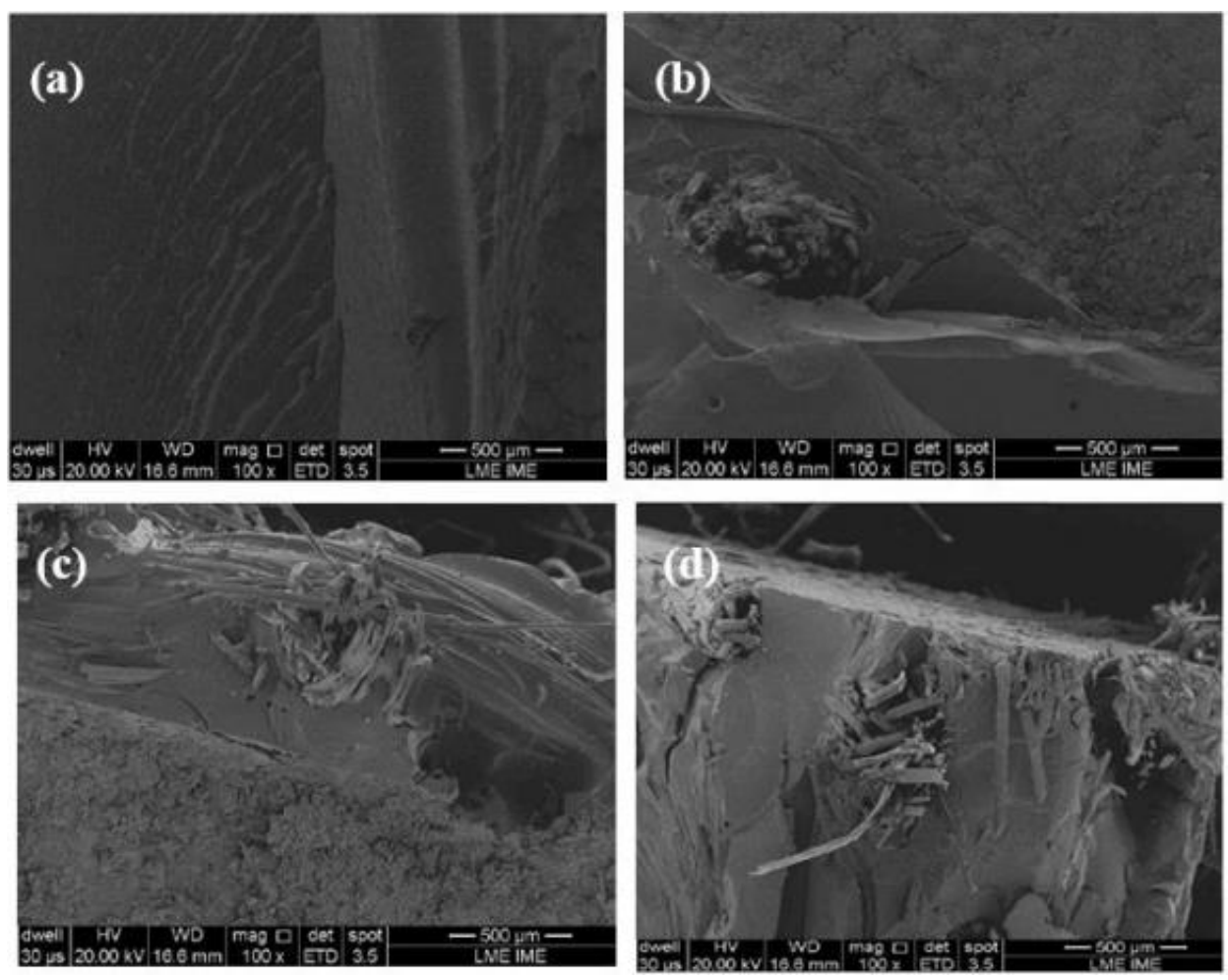

Figura 6: Superfície de fratura de impacto Charpy da amostra de epóxi puro (a) e compósito de epóxi reforçado com 16,70vol\% (b), 21,84vol\% (c) e 32,30vol\% de tecido de fique (d).

Este mecanismo de fratura que, trincas se propagam preferencialmente entre os tecidos de fique e de superfície da matriz de epóxi devido à baixa tensão interfacial. A área de fratura associado como o tecido de fique que atuam como reforço para o compósito, justifica a maior energia de impacto absorvido com aumento crescente da quantidade de fibras de fique.

\section{CONCLUSÃO}

- A incorporação de tecido de Fique aumenta a resistência ao impacto de compósitos de matriz epóxi em comparação com resina pura, o que mostra que o tecido de Fique é um bom material de reforço para impactar cargas.

- A resistência ao impacto aumenta linearmente com a incorporação de tecido de fique até $32,30 \mathrm{vol} \%$ em volume.

- Junto com a retenção de algumas fibras do tecido de fique inteiras após impacto, a baixa resistência interfacial resulta em maior absorção de energia devido à propagação de trincas na interface fibra / matriz, permitindo a formação de uma maior área de fratura longitudinal em relação a uma fratura transversal, o que deve ocorrer na matriz para romper as fibras

\section{REFERÊNCIAS}


[1] Callister Jr., WD. Ciência e engenharia de materiais: uma introdução: LTC, 2002, p 368

[2] Wambua P, Ivens I and Verpoest I. Composites Science and Technology Vol. 63 (2003), p. 1259.

[3] Marsh G. Mater. Next step for automotive materials. Today, v. 6(4) (2003), p. 36.

[4] [Holbery J, Houston D, Natural-fiber-reinforced polymer composites in automotive applications, JOM 2006, p. 80-6.

[5] Zah R, Hischier R, Leão and I. Braun. Curaua fibers in the automobile industry - A sustainability assessment. J. Cleaner Production, 15 (2007) 1032-1040.

[6] Crocker J, Natural materials innovative natural composites, Materials Technology, 2008, p. 174-8.

[7] Monteiro SN, Lopes FPD, Ferreira AS, Nascimento DCO, Natural fiber polymer matrix composites: cheaper, tougher and environmentally friendly, JOM 2009, p. 17.

[8] Joshi SV, Drzal LT, Mohanty AK, Arora S, Are natural fiber composites environmentally superior to glass fiber reinforced composites, Compos. Part A, 2004, p. 3716.

[9] Kalia S, Kaith BS, Kaurs I. Cellulose Fibers: Bio - and Nano-Polymer Composites.( New York: Springer, 2011).

[10] Montoya A, Montoya I, Castellanos O. Propuesta de gestión ambiental em sistemas de cadena productiva. Caso: cadena de fique em colombia, In: congresso Anual de la academia de ciências administrativas, ACACIA, Impacto de los câmbios estratégicos em el entorno y em las organizaciones Acapulco, 2004: p. 10

[11]ASTM - American Society for Testing Materials. ASTM D6110 - 04 Standard Test Methods for Determining the Charpy Pendulum Impact Resistance of Notched Specimens of Plastics. Annual Book of ASTM, 2004

[12] Mohanty AK, Misra M, Hinrichsen G, Biofiber, Biodegradable polymers and biocomposites: an overview. Macromol Mat Eng, 2000 p. 1-24. 